\title{
Improved Power Quality Monitoring through Phasor Measurement Unit Data Interpretation
}

Pertl, Michael; Marinelli, Mattia; Bindner, Henrik W.

Published in:

Proceedings of Power Engineering Conference (UPEC) 2015

Publication date:

2015

Document Version

Publisher's PDF, also known as Version of record

Link back to DTU Orbit

Citation (APA):

Pertl, M., Marinelli, M., \& Bindner, H. W. (2015). Improved Power Quality Monitoring through Phasor

Measurement Unit Data Interpretation. In Proceedings of Power Engineering Conference (UPEC) 2015 IEEE.

\section{General rights}

Copyright and moral rights for the publications made accessible in the public portal are retained by the authors and/or other copyright owners and it is a condition of accessing publications that users recognise and abide by the legal requirements associated with these rights.

- Users may download and print one copy of any publication from the public portal for the purpose of private study or research.

- You may not further distribute the material or use it for any profit-making activity or commercial gain

- You may freely distribute the URL identifying the publication in the public portal

If you believe that this document breaches copyright please contact us providing details, and we will remove access to the work immediately and investigate your claim. 


\title{
Improved Power Quality Monitoring through Phasor Measurement Unit Data Interpretation
}

\author{
Michael Pertl, Mattia Marinelli, Henrik Bindner \\ Department of Electrical Engineering (Center for Electric Power and Energy) \\ DTU - Technical University of Denmark \\ Contact: mpertl@elektro.dtu.dk
}

\begin{abstract}
The observability needs in future power systems will change radically due to the continuing implementation of renewable energy sources at all voltage levels. Especially in distribution grids new observables will be needed in order to monitor the state of the power system sufficiently and to perform the correct actions for operating the system. In future power systems more measuring sensors including phasor measurement units will be available distributed all over the power system. They can and should be utilized to increase the observability of the power system. In this paper the impact of photovoltaic and wind power production on the voltage unbalance was analyzed. PMU data and NTP-synchronized data from two different MV networks were used. It has been found that PV production has only a minor negative impact on the voltage unbalance whereas the wind power production has a great positive impact. The voltage unbalance factor (VUF) could be a 'new' observable for a particular power system condition. Information about the actual injected wind power for a certain grid area could be derived without knowing/measuring the real wind power injection.
\end{abstract}

Index Terms-- Phasor Measurement Unit, Distribution Grid, Voltage Unbalance Factor, Photovoltaic, Wind Power

\section{INTRODUCTION}

Phasor measurement units (PMU) are commonly used in transmission grids and rarely in distribution grids. In fact, the observability of transmission grids is much better compared to distribution grids, which means, the observability decreases with decreasing voltage level of the grid. Considering that renewable distributed sources are progressively installed at distribution level, challenges arise when it comes to optimal utilization of distribution networks. By 2030, the system will be changed from a centralized to a distributed system, with generation and demand units distributed all over the electric power system, at all voltage levels.

The EU FP7 project ELECTRA (electrairp.eu) addresses the challenges for the future 2030+ power system with deployment of RES across all voltage levels. One of the work packages called 'Increased Observability' deals with the observability of the power system and the first findings regarding future observables and observability needs and are published in [1] and [2]. The two papers point out the crucial need to evolve and improve the present monitoring/observing schemes as well as introducing new observables to develop new control schemes and improve the existing ones.

According to the report on Integration of Renewable Energy in Europe in 2050 the share of generation from RES will be in a range from 51 to $68 \%$ [3]. A considerable amount of renewable generation units such as photovoltaics (PV) are connected through power electronic devices which can lead into power quality issues such as harmonic pollution [4]. Furthermore, single phase loads/generators (e.g. PV inverters) negatively impact the voltage and current unbalance in the power system. Therefore the power quality has to be monitored in real time to identify critical issues and resolve them as fast as possible. For instance, the VUF could be utilized to derive information about the present system state.

The aim of the paper is to study the power quality with emphasis on voltage unbalance and how it is affected by high penetration of PV and wind power. Since the voltage unbalance factor is a very important power quality parameter it should be monitored carefully, because great voltage unbalances result in great unwanted neutral/ground currents or stress for sensitive electrical equipment or even grid disconnection of inverter based devices. Further information about impact of PV and wind power on the power quality can be found in [5]-[7].

For the analysis historical data from two different power systems were used. Firstly, PMU data from the smart grid project at EPFL [8] in Switzerland was used. One of the peculiarities of the network is the high amount of installed PV of $2 \mathrm{MW}$ in their medium voltage network $(20 \mathrm{kV})$. The PMU data from past measurements of the project is provided online at smartgrid.epfl.ch. Secondly, measurement data from the Danish island Bornholm, where a great amount of wind power is integrated into the distribution system was used for the analysis. The installed wind power adds up to $30 \mathrm{MW}$, whereas the peak load equals to around $56 \mathrm{MW}$. Further information about the power system from Bornholm can be found in [9]. The measurement data from Bornholm is not measured by PMUs, but nevertheless, they got measured synchronized with the network time protocol (NTP), which is not as accurate as GPS synchronization but sufficient for the analysis carried out. Differences and/or similarities between the different power systems are pointed out and discussed.

The voltage unbalance limit for distribution systems is regulated by EN-50160 and is set to one percent for high voltage level, measured as 10-minute values [10], [11].

The paper is organized as follows. In section II the synchrophasor technology is explained and the calculation for the complex voltage unbalance factor (CVUF) and the symmetrical components are set out. Section III gives the two analysed grids and detailed information about their 
peculiarities. The results are presented in section IV and concluded in section $\mathrm{V}$.

\section{METHODOLOGY}

\section{A. Introduction to Synchrophasor Technology}

Synchrophasor technology uses the global positioning system (GPS) to generate time synchronous measurements of voltage and current at different locations in an electrical power system. The voltage and current is measured as a phasor which consists of magnitude and angle. The synchronized measurement at different locations in the network offers new technical opportunities compared to conventional local measurements, e.g. voltage angle at different locations of the network, so also the voltage angle difference between two measuring points. In the simplest setup the measurement system consists of a phasor measurement unit (PMU), a phasor data concentrator (PDC) and an evaluation software to process the measured data, e.g. for real time monitoring of the power system. The PMU is the measuring unit which is placed at certain points in the network and actually makes the measurement. The measurement data from several PMUs is then sent to the PDC which stores the data and provides it for the evaluation software or for post event analysis (off-line analysis). The requirements for synchronized measurements, such as data formats, communication protocol, synchronization requirements, error limits, etc. are specified by standards. In [12] the development of the IEEE C37.118 and IEC 61850 standards are explained. The complete detailed description of the IEEE C37.118 standard is stated in [13].

\section{B. Definition of synchrophasors}

The synchrophasor representation $\mathrm{X}$ of a signal $\mathrm{x}(\mathrm{t})$ is given by:

$$
\begin{gathered}
X=X_{r}+j X_{i} \\
=\frac{X_{m}}{\sqrt{2}} \cdot e^{j \phi} \\
=\frac{X_{m}}{\sqrt{2}} \cdot(\cos \phi+j \sin \phi)
\end{gathered}
$$

$\mathrm{X}_{r}, X_{i} \quad$ real and imaginary part of the signal $\mathrm{x}(\mathrm{t})$

$\frac{X_{m}}{\sqrt{2}} \quad$ rms value of the signal $\mathrm{x}(\mathrm{t})$

instantaneous phase angle relative to cosine function at nominal system frequency synchronized to universal time coordinated (UTC)

\section{Angle of the phasor}

Since the measurement system compares the signal angle with the synchronized reference cosine signal at nominal frequency it is clear that the angle of the measured signal would not change if the frequency of the measured signal matches the nominal frequency. In the case the frequency of the measured signal deviates from the nominal frequency the angle changes uniformly at a rate of $2 \pi\left(f-f_{0}\right)$ with $T_{0}=1 / f_{0}$ [13]. That means that the phasor is rotating in the complex plane. This kind of phase angle is better known as absolute angle which makes the measured angles at different locations comparable with each other. Although the angle of the measured signal is changing at non nominal frequency the magnitude remains unchanged as long as the amplitude of the measured signal is constant. Fortunately, the angle itself is not very much from interest instead the phase angle between two different things is interesting, e.g. the voltage angle between two different locations or the angle between voltage and current. A very deep and detailed explanation can be found in the white paper [14] which is provided by the manufacturer of the used PMU model. Fig. 1 shows how a PMU is measuring the phasors. The dashed line represents the GPS-synchronized reference signal whereas the solid line shows the signal being measured.

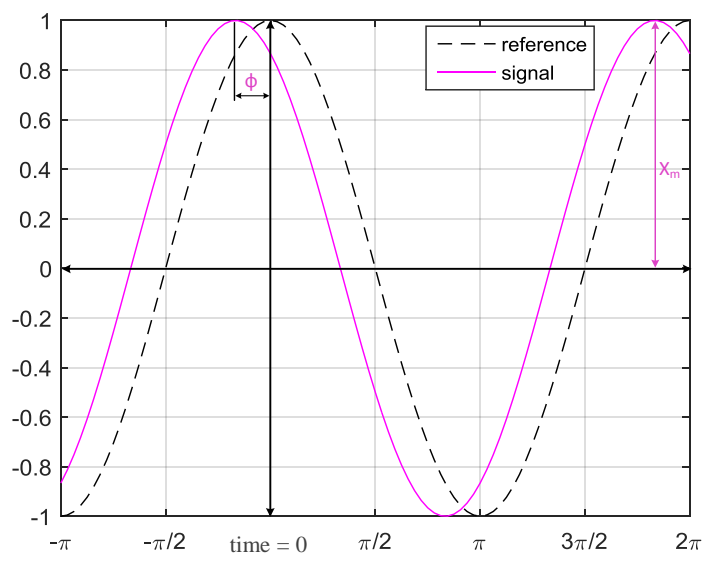

Fig. 1 - Synchrophasor angle measurement

\section{Definition of slack angle}

As mentioned before, the angle from voltage and current is given as absolute angle which changes over time. Since all the signals in the same system have the same frequency it is meaningful to set one angle as a reference (slack) and subtract this angle from all the others. In this case the voltage angle from phase ' $a$ ' was set as reference angle, so this angle got subtracted from all the other angles. This operation has the effect that all the angles get independent from the frequency.

\section{E. Definition of the voltage and current unbalance}

Since there are different approaches for the calculation of the unbalance (see [15]) the used one has to be explained. In this paper the complex unbalance factor is expressed as CVUF for voltage and CCUF for the current unbalance. The value is given in percent and is calculated as expressed by the International Electrotechnical Commission (IEC): 


$$
\begin{gathered}
C V U F=\frac{U_{N}}{\frac{U_{P}}{I_{P}}} \cdot 100 \% \\
\text { CCUF }=\frac{I_{N}}{I_{P}} \cdot 100 \%
\end{gathered}
$$

Where $\underline{U}_{N}$ and $\underline{I}_{N}$ are the complex negative-sequence voltages and currents and $\underline{U}_{P}$ and $I_{P}$ is the complex positivesequence voltages and currents, respectively. In this paper the current unbalance factor was not used, but it is given here to be complete.

\section{F. Calculation of symmetric components}

In the following the calculation of the symmetric components is given, which is the same for voltage and current and is calculated via the established transformation below.

$$
\underline{U}^{S}=\underline{S} \cdot \underline{U}_{p}=\left[\begin{array}{l}
\underline{U}^{0} \\
\underline{U}^{1} \\
\underline{U}^{2}
\end{array}\right]=\frac{1}{3}\left[\begin{array}{ccc}
1 & 1 & 1 \\
1 & \underline{a} & \underline{a}^{2} \\
1 & \underline{a}^{2} & \underline{a}
\end{array}\right]\left[\begin{array}{l}
\underline{U}_{1} \\
\underline{U}_{2} \\
\underline{U}_{3}
\end{array}\right]
$$

$$
\begin{array}{cl}
\underline{U}^{S} & \text { vector containing symmetrical components } \\
\underline{S} & \text { transformation matrix } \\
\underline{U}_{p} & \text { vector containing phase voltage phasors } \\
\underline{U}^{0}, \underline{U}^{1}, \underline{U}^{2} & \text { symmetric components (zero-, positive-, } \\
\underline{U}_{1}, \underline{U}_{2}, \underline{U}_{3} & \text { negative-sequence voltage) } \\
\text { a } & \text { phase voltage phasors } \\
& \text { phase shift of } 120^{\circ}\left(=e^{j \cdot \frac{2 \pi}{3}}\right)
\end{array}
$$

Once the complex symmetric components are calculated the complex voltage CVUF and current unbalance factor CCUF can be calculated as in (2) and (3).

\section{ANALYZED GRIDS}

The analysis was carried out for two different grids where both of them have their own peculiarities. One of them has a great amount of PV installed whereas the other one is wind dominated.

\section{A. EPFL Smart Grid}

The École polytechnique fédérale de Lausanne (EPFL) has established a smart grid project on the University campus which provides a particularly challenging $20 \mathrm{kV}$ medium voltage distribution grid (see Fig. 2) for research in active distribution networks (ADNs). The so called smart grid includes measurements from five PMUs distributed over the network. Moreover, these PMU measurements are made available on the website smartgrid.epfl.ch and can be downloaded by anyone.

For the analysis within this paper the installed capacity of 2 MW of photovoltaic panels (three phase inverter) was from interest with respect to the impact on the voltage unbalance in the network. The blue dots show the locations where PMUs are installed.
The data for the analysis in this paper originates from the PMU at location which is called 1.PC-2. At this point the EPFL grid is connected to the external grid where the PMU is connected on the medium voltage side of the transformer.

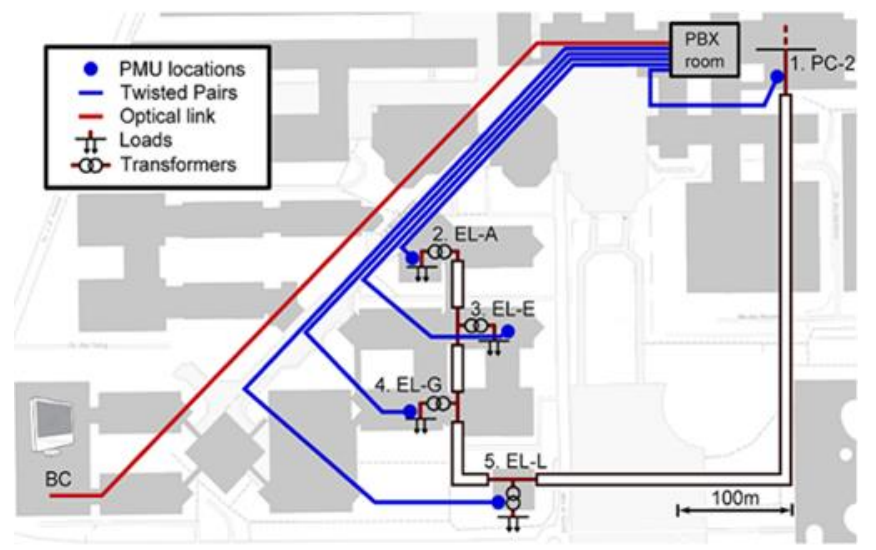

Fig. 2 - EPFL Smart Grid at University campus in Lausanne [8]

\section{B. Bornholm Power System}

Bornholm is a small Danish island which is located south of Sweden. The Bornholm power system is a distribution system with around 28000 customers and a peak load of $56 \mathrm{MW}$ which is electrically only connected to Sweden via a $60-\mathrm{kV}$-cable with a capacity of $60 \mathrm{MW}$ [16]. Further information about the Bornholm power system can be found in [9].

One interesting aspect for the analysis was that the installed wind power on the island is around $30 \mathrm{MW}$, which is about 54 $\%$ of the peak load. So, with respect to the energy mix it can be said that the electric power production is highly dominated by wind power during high wind periods.

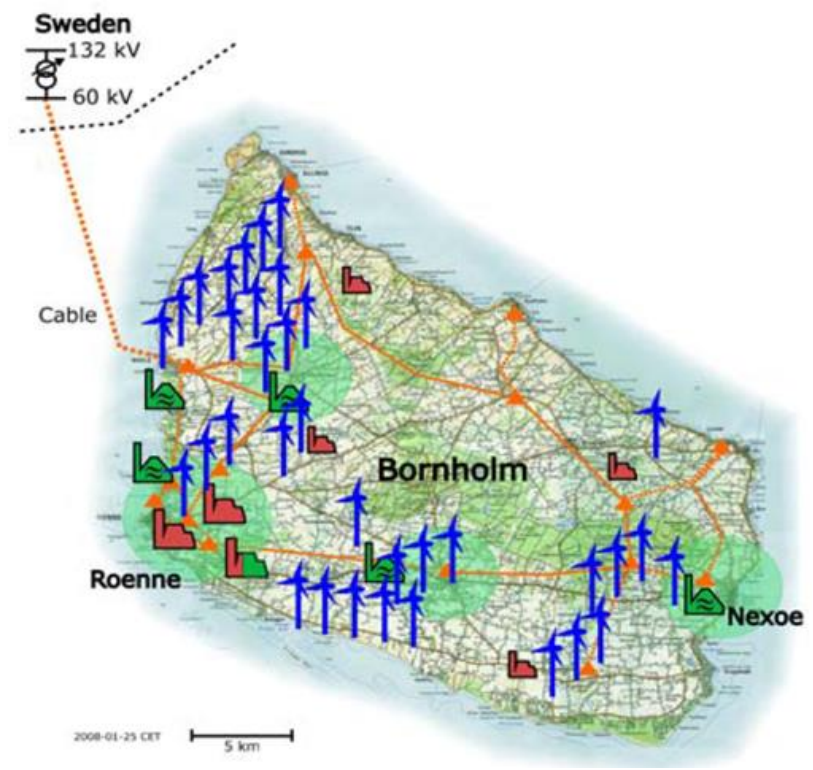

Fig. 3 - The Bornholm power system [9] 
For the analysis measurements of the voltage unbalance from the Feeder Borrby at the Substation Hasle was used. The substation is located at the incoming sea cable from Sweden. So it is the main (only) connection point to an external grid. Measurements for the wind power production on the island were taken from the wind park Aakirkeby which is connected to the feeder Kalby. Electrically, the two measuring locations are about $30 \mathrm{~km}$ from each other. The wind power production of this wind park was used as a representative for the whole wind power production on the island. Since the island is small, this assumption is acceptable (see Fig. 4).

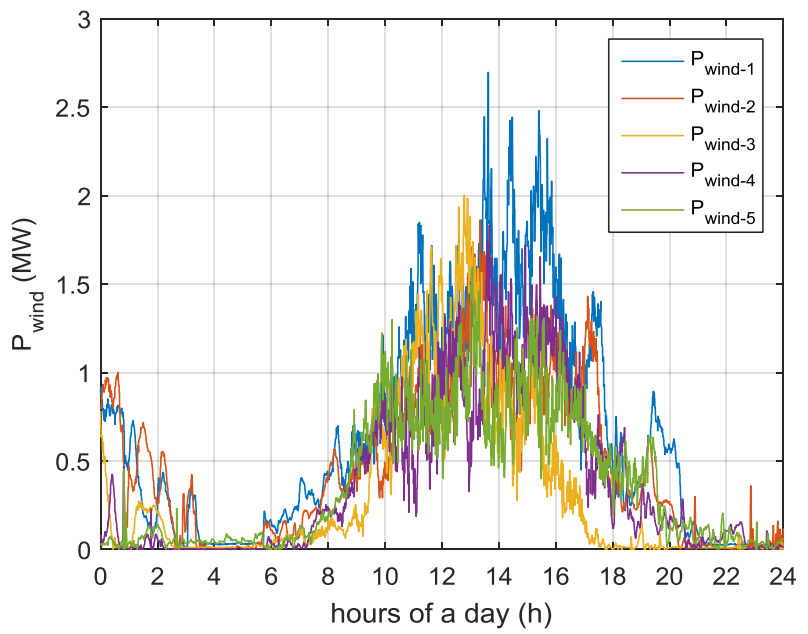

Fig. 4 - Wind power production from different wind parks for a particular day at the island of Bornholm

\section{RESULTS}

In this section the results from the analysis are presented and described.

The first analysis dealt with the impact from PV production on the voltage unbalance factor. This study was carried out with the data from the EPFL grid, because there is a great amount of PV (2 MW) installed in the grid.

The second analysis dealt with the impact from wind power production on the voltage unbalance factor. This study was carried out with data available from the Danish island Bornholm where $30 \mathrm{MW}$ of wind power is installed in a grid with $56 \mathrm{MW}$ peak load.

\section{Scenario I - EPFL Smart Grid with PV penetration}

Fig. 5 and Fig. 6 respectively show the time series of the VUF for one particular summer and winter day which has been calculated out of the data from the PMU in the EPFL grid. The red curves show the averaged Surface Incident Shortwave Flux (SISF) for the area of Lausanne for the same days respectively. The solar radiation data originates from the NASA Goddard Earth Sciences (GES) Data and Information Service Center (DISC). It can be accessed through the so called Mirandor system on the webpage mirador.gsfc.nasa.gov. [17]
As it can be seen clearly in Fig. 5, the solar radiation begins to increase in the morning and reaches the peak at about 9 o'clock. It is expected that the peak would be reached around midday, but the first guess is a cloudy condition during midday and early afternoon which would explain the trend. It has to be mentioned that, this is an assumption and has not been proofed, but it does not affect the analysis carried out here. After reaching the peak it begins to decrease and finally reaches the initial value of zero at about $7 \mathrm{pm}$.

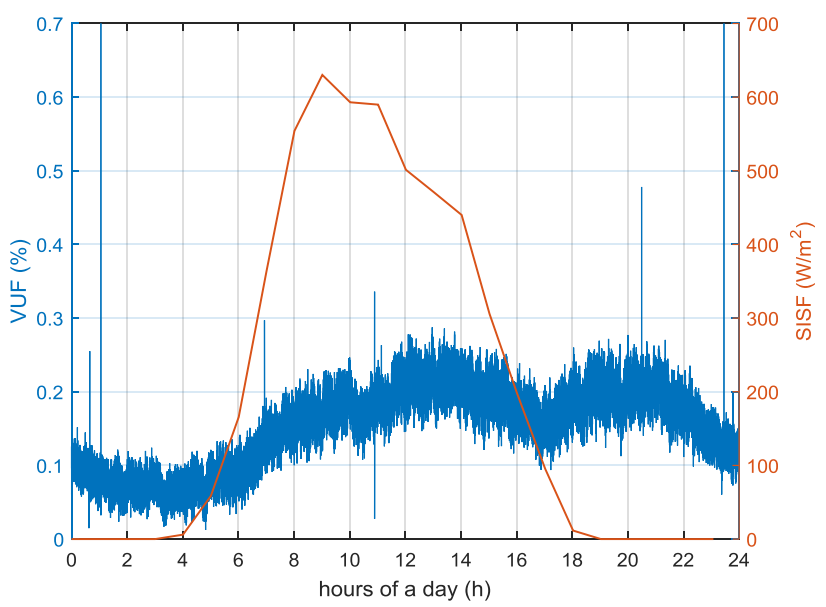

Fig. 5 - Voltage Unbalance Factor measured at MV interface of EPFL Smart Grid and Surface Incident Shortwave Flux for a summer day

In Fig. 6 can be seen that the VUF starts to increase at around 8 am and reaches its peak at about $1 \mathrm{pm}$. Then it starts again to decrease and finally there is, like in the previous case, another peak in the evening visible, but it is smaller compared to the summer case. The peak in the evening could be caused by some single phase appliances, but it cannot be determined due to lack of measurements/information about the load situation. As it can be seen clearly, the solar radiation in winter is much smaller than in summer.

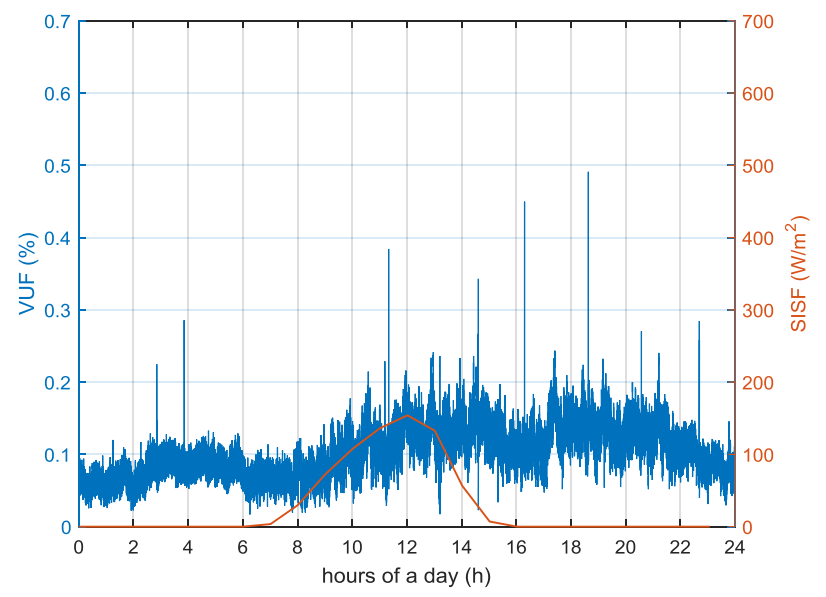

Fig. 6 - Voltage Unbalance Factor measured at MV interface of EPFL Smart Grid and Surface Incident Shortwave Flux for a winter day 


\section{Scenario II - Bornholm Power System with wind penetration}

Fig. 7 and Fig. 8 depict the VUF (blue line) at the substation Hasle, feeder Borrby which is the substation where the sea cable from Sweden comes in. Additionally, the associated wind power production (green line) from the wind park Aakirkeby is illustrated. Both plots are assigned to different days.

The different depiction of the two plots is simply because of a better visualization for the reader. In Fig. 8 the two lines would overlap too much and therefore it is plotted separately whereas this is not necessary in Fig. 7.

Both, Fig. 7 and Fig. 8 are intended to show a change in wind power production during the hours of two particular days on the island of Bornholm. As mentioned before, the green line shows the wind power production of a wind park which is connected to the distribution system at the substation Aakirkeby. The chosen wind park should be the representative for the wind power generation on the whole island in order to reduce the data for the analysis and to simplify the plots. Since the island is a quite small island the assumption of one representative wind park is arguable as the wind power production for one particular day is depicted in Fig. 4. It is clearly visible that the wind power production from different wind parks is strongly aligned with each other. For simplicity the different wind parks are labelled with numbers one to five. However, they are distributed across the whole island.

The blue line in the plots show the VUF at the substation Hasle where the sea cable from Sweden is connected to the Bornholm power system. That means that the measuring location for the VUF differs from the measuring location from the wind power production. That shows that the impact of the wind power is not a local issue, but it is a network wide phenomenon.

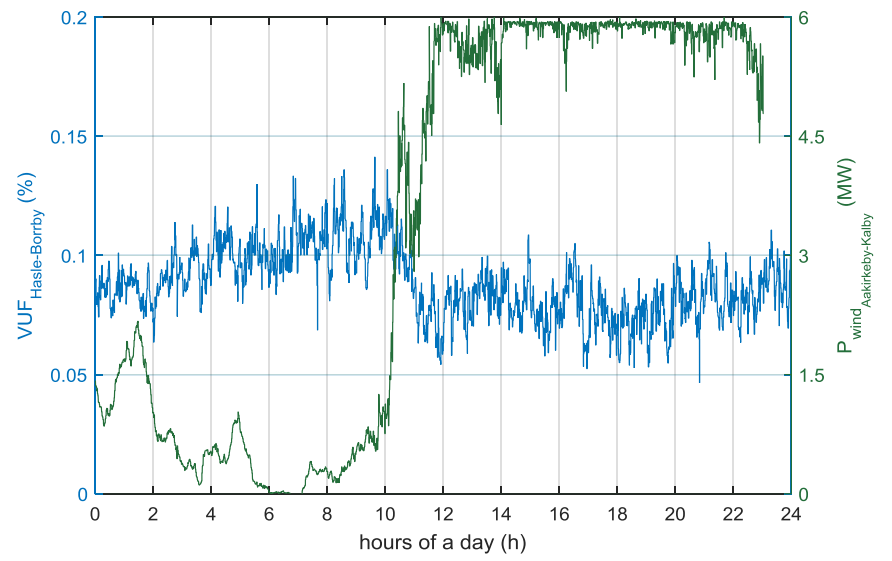

Fig. 7 - Voltage Unbalance Factor (Hasle-Borrby) and Wind Power Generation (wind park Aakirkeby-Kalby) for one particular day at Bornholm

In Fig. 7 the wind power generation starts to increase at around 10 am and maintain very high for the following hours.
The VUF has a slight increase over the whole morning and then drops to a lower value as soon as the wind production begins to increase.

Fig. 8 shows a different example with wind power production from Bornholm where the wind power generation increases during midday and decreases afterwards. The VUF shows the same characteristics as in the case before, namely a decreased value during high wind power generation, but in this example it can also be seen that the VUF again increases as the wind power production reduces.
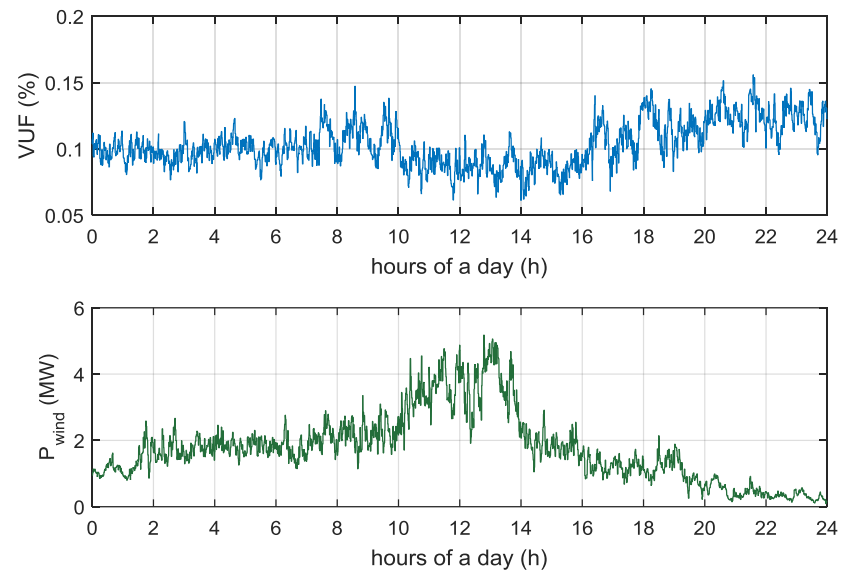

Fig. 8 - Voltage Unbalance Factor (Hasle-Borrby) and Wind Power Generation (wind park Aakirkeby-Kalby) for another day at Bornholm

To emphasize the visual results a calculation of the correlation for the two different cases was carried out and is presented in Table I. The correlation between the surface incident shortwave flux/wind power generation and the VUF was calculated with Matlab using the linear Pearson correlation. Further information about correlation can be found in reference [18].

The results from the correlation calculation confirm the conclusions from the visual examination. As it can be seen in Table I the results for scenario I and II differ with respect to the plus/minus sign. The correlation in the PV scenario has a positive correlation whereas the wind scenario has a negative one. That means that PV has a negative impact on the VUF and the wind power production a positive effect. It can also be seen that the correlation factor for both wind power cases is much higher compared to the both PV cases. Moreover, the correlation shows in both scenarios higher values for the summer day than for the winter day.

TABLE I - CORRELATION RESULTS FOR BOTH SCENARIOS

\begin{tabular}{|c||c|c|}
\hline \multicolumn{1}{|c||}{} & \multicolumn{2}{c|}{ Correlation } \\
\hline \hline Scenario & summer day & winter day \\
\hline I (PV) & 0.4292 & 0.2237 \\
\hline II (wind) & -0.6692 & -0.5754 \\
\hline
\end{tabular}




\section{CONCLUSION AND FUTURE WORK}

The paper shows that there is a relation between different renewable energy resources connected to the grid and the voltage unbalance factor, which is a very important power quality parameter since it may lead to consistent undesired neutral/ground currents or stress for sensitive electrical equipment or even grid disconnection of inverter based devices. In case of the relation between the photovoltaic production and the voltage unbalance it can be said that the impact of the generated solar power has a rather small impact on the voltage unbalance factor as it can be seen from Table I and Fig. 5 and Fig. 6. Nevertheless, at least a small impact of the PV power production on the VUF can be attested. When comparing Fig. 5 and Fig. 6 it is visible that the VUF during the summer day has greater increase than during the winter day. Both figures show a peak in the evening which obviously cannot be caused by PV generation, so it has to be caused by other sources. Since the impact from PV production on the VUF is very small the problems of high PV penetration at LV- and MV-level is rather linked to other issues, e.g. voltage violations due to high PV penetration. In case of a lot of small single-phase connected PV production it could perhaps lead into voltage unbalance issues, but three-phase inverters are rather unproblematic regarding voltage unbalance issues. However, the impact of wind power production on the VUF can be classified as relatively high as it can be seen in Fig. 7 and Fig. 8. The VUF has a negative relation with the VUF in the concerned power system, i.e. when the wind power production increases the VUF decreases and vice versa. This visual examination of the plots has also been confirmed by calculating the correlation factor between the VUF and the injected wind power. From the results of the calculation in Table I can be derived that the wind power injection has a fairly high positive impact on the VUF since it decreases while high wind penetration. However, there are several other parameters and uncertainties which affecting the VUF which have not been taken into account in this studies. In summary it can be concluded that the continuing integration of RES always affects different power quality parameters. This impact may not always lead to worsening of system parameters, as shown in the wind power analysis. Furthermore, monitoring the VUF could give useful information about the power system state with respect to RES production in a certain grid area without knowing all the specific measurements from RES connected to this part of the grid.

\section{ACKNOWLEDGEMENTS}

Michael Pertl is a PhD student at the Technical University of Denmark (DTU) and is supported by the EU FP7 project ELECTRA (grant: 609687) and the Danish Research Project "ELECTRA Top-up" (grant: 3594756936313). More information at electrairp.eu.

\section{REFERENCES}

[1] A. Z. Morch, S. H. Jakobsson, K. Visscher, and M. Marinelli, "Future control architecture and emerging observability needs," in Power Engineering, Energy and Electrical Drives (POWERENG), 2015.

[2] K. Visscher, M. Marinelli, A. Z. Morch, and S. H. Jakobsson, "Identification of observables for future grids - the framework developed in the ELECTRA project," in PowerTech, 2015.

[3] European Commission, "Integration of Renewable Energy in Europe," 2014.

[4] E. Caamaño-Martín, H. Laukamp, M. Jantsch, T. Erge, J. Thornycroft, H. De Moor, S. Cobben, D. Suna, and B. Gaiddon, "Interaction between photovoltaic distributed generation and electricity networks," Prog. Photovoltaics Res. Appl., vol. 16, no. 7, pp. 629-643, 2008.

[5] National Renewable Energy Laboratory (NREL), "DG Power Quality , Protection and Reliability Case Studies Report DG Power Quality , Protection and Reliability Case Studies Report (Technical Report)," 2003.

[6] E. Caamano, J. Thornycroft, H. De Moor, and S. Cobben, "State-of-theArt on Dispersed PV Power Generation: Publications review on the impacts of PV Distributed Generation and Electricity networks (Technical Report)," 2007.

[7] M. G. Sugirtha and P. Latha, "Analysis of power quality problems in grid connected wind power plant," Int. Conf. Recent Adv. Electr. Electron. Control Eng., pp. 19-24, 2011.

[8] M. Pignati, M. Popovic, S. Barreto, R. Cherkaoui, G. D. Flores, J. Le Boudec, M. Paolone, P. Romano, S. Sarri, T. Tesfay, D. Tomozei, and L. Zanni, "Real-Time State Estimation of the EPFL-Campus MediumVoltage Grid by Using PMUs," in 6th IEEE PES International Conference and Exhibition on Innovative Smart Grid Technologies, 2015.

[9] J. Østergaard and J. Nielsen, "The Bornholm Power System An Overview (Technical Report)," Denmark, 2008.

[10] J. Driesen and T. Van Craenenbroeck, "Voltage Disturbances: Introduction to Unbalance," Copp. Dev. Assoc., 2002.

[11] H. Markiewicz and A. Klajn, "Voltage Disturbances," Power Qual. Appl. Guid., vol. 5.4.2, pp. 4-11, 2004.

[12] K. E. Martin, "Synchrophasor standards development - IEEE C37.118 \& IEC 61850," Proc. Annu. Hawaii Int. Conf. Syst. Sci., pp. 1-8, 2011.

[13] IEEE Power Engineering Society, "IEEE standard for synchrophasors for power systems - IEEE Std C37.118-2005," IEEE Power Eng. Soc., pp. 1$65,2005$.

[14] I. Arbiter Systems, "What is Absolute Phase Angle?" [Online]. Available: http://www.arbiter.com/files/product-attachments/absolute_phase.pdf. [Accessed: 20-Jan-2015].

[15] P. G. Kini, R. C. Bansal, and R. S. Aithal, "A Novel Approach Toward Interpretation and Application of Voltage Unbalance Factor," in IEEE Transactions on Industrial Electronics, 2007, vol. 54, no. 4, pp. 23152322.

[16] E. James-Smith and M. Togeby, "Security of supply for Bornholm Integration of fluctuating generation using coordinated con- Demand side options for system reserves (Technical Report)," Copenhagen, 2007.

[17] "Global Modeling and Assimilation Office (GMAO) (2007),MERRA 2D IAU Diagnostic, Radiation Surface and TOA, Time Average 1-hourly (2/3x1/2L1),version 5.2.0, NASA Goddard Space Flight Center:, Accessed April 9th 2015 Date at http://disc.sci.gsfc.nasa." .

[18] M. G. Kendall and J. D. Gibbons, "Rank Correlation Methods," Sci. Forum, vol. 3, p. 260, 1990. 